\title{
SIMPLE COMPACTIFICATIONS AND POLAR DECOMPOSITION OF HOMOGENEOUS REAL SPHERICAL SPACES
}

\author{
FRIEDRICH KNOP \\ Department Mathematik, Emmy-Noether-Zentrum \\ FAU Erlangen-Nürnberg, Cauerstr. 11, 91058 Erlangen, Germany \\ BERNHARD KRÖTZ \\ Universität Paderborn, Institut für Mathematik \\ Warburger Straße 100, D-33098 Paderborn, Germany \\ EITAN SAYAG \\ Department of Mathematics, Ben Gurion University of the Negev \\ P.O.B. 653, Be'er Sheva 84105 , Israel

\begin{abstract}
HENRIK SCHLICHTKRULL
University of Copenhagen, Department of Mathematics

Universitetsparken 5, DK-2100 Copenhagen Ø, Denmark
\end{abstract}

\begin{abstract}
Let $Z$ be an algebraic homogeneous space $Z=G / H$ attached to real reductive Lie group $G$. We assume that $Z$ is real spherical, i.e., minimal parabolic subgroups have open orbits on $Z$. For such spaces we investigate their large scale geometry and provide a polar decomposition. This is obtained from the existence of simple compactifications of $Z$ which is established in this paper.
\end{abstract}

\footnotetext{
E-mail addresses: friedrich.knop@fau.de, bkroetz@math.uni-paderborn.de, eitan.sayag@gmail.com, schlicht@math.ku.dk.

Date: May 29, 2014.

2000 Mathematics Subject Classification. 14M27, 22F30, 22E15.

Key words and phrases. spherical space, polar decomposition.

The second named author was supported by ERC Advanced Investigators Grant HARG 268105.
} 


\section{INTRODUCTION}

Our concern is the large scale geometry of algebraic homogeneous spaces $Z=G / H$ attached to an algebraic real reductive group $G$. Here $H<G$ is an algebraic subgroup.

One approach towards the large scale geometry of $Z$ is to study the double-coset space $K \backslash G / H$ for $K<G$ a maximal compact subgroup. In interesting cases, for example if $H$ is a symmetric subgroup which is compatible with the choice of $K$, i.e., $H \cap K$ is maximal compact in $H$, then there is a good answer in terms of the generalized Cartan decomposition for $Z=G / H$ : there is a non-compact torus $A_{q}$ with Lie algebra orthogonal to $\mathfrak{h}+\mathfrak{k}$ such that $G=K A_{q} H$. Here $\mathfrak{h}$ and $\mathfrak{k}$ denote the Lie algebras of $H$ and $K$.

The class of homogeneous spaces $Z$ we consider in this paper are those which are called real spherical, i.e., minimal parabolic subgroups of $P$ admit open orbits. Symmetric spaces are real spherical and basic properties of symmetric spaces have been shown to persist in the larger class of real spherical spaces (see [1], [13], [14], [12], [15]).

The objective of this paper is to study the large scale geometry of real spherical spaces. In the past we looked at many non-symmetric examples and constructed non-compact tori a such that $G=K A H$ holds true, but could not find a general construction scheme, see [13], 6]. In contrast to symmetric spaces the tori $\mathfrak{a}$ are typically not orthogonal to $\mathfrak{k}+\mathfrak{h}$ which makes matters rather complicated.

In order to discuss the large scale geometry of spherical spaces it thus seems reasonable to weaken the concept of the polar decomposition $G=K A H$ and replace $K$ by a compact subset $\Omega \subset G$. This approach is motivated by the investigations in [16] for a class of p-adic spherical spaces.

By definition a minimal parabolic subgroup $P<G$ is given by $P=$ $G \cap P_{\mathbb{C}}$ where $P_{\mathbb{C}}<G_{\mathbb{C}}$ is a minimal parabolic of $G_{\mathbb{C}}$ which is defined over $\mathbb{R}$. Here $G_{\mathbb{C}}$ denotes the complexification of $G$ which is a complex reductive algebraic group.

One main geometric result of this paper then is:

Theorem 1.1. [Polar Decomposition] Let $Z=G / H$ be a real spherical space attached to an algebraic real reductive group. Let $P$ be a minimal parabolic subgroup of $G$ such that $P H$ is open. Then there is a Levi decomposition $P=M A \ltimes N$ such that

$$
G=\Omega A F H
$$

for a compact set $\Omega \subset G$ and a finite set $F \subset G$. 
The main new tool for deriving the polar decomposition is the existence of a simple compactification. The definition is given in Section 3 , Most importantly, a simple compactification has a unique closed orbit, and thus the following result is obtained in Section 3.3 .

Theorem 1.2. Let $Z=G / H$ be a real spherical space and assume that $H$ is equal to its normalizer, $H=N_{G}(H)$. Let the subgroup $J<G$ be defined by

$$
J=\left\{g \in G \mid P_{\mathbb{C}} H_{\mathbb{C}} g=P_{\mathbb{C}} H_{\mathbb{C}}\right\} .
$$

Then there exists a compact subgroup $M_{J}$ of $J$ such that $J=M_{J} H$. In particular, $\mathrm{J} / \mathrm{H}$ is compact.

Furthermore, there exists an irreducible rational real representation $V$ of $G$ with $J$-fixed vector $v_{J}$ such that

$$
Z_{J}:=G / J \rightarrow \mathbb{P}(V), \quad g H \mapsto\left[g \cdot v_{J}\right]
$$

is an embedding, and such that the closure of $Z_{J}$ in $\mathbb{P}(V)$ is a $G$ compactification of $Z_{J}$ with a unique closed $G$-orbit.

The proof of Theorem 1.1 follows in Section 4. In Section 5 we define the compression (or valuation) cone of $Z$. We show that the compression cone governs the fine convex geometry near the closed orbit of a simple compactification and results in a refined polar decomposition, Theorem 5.13. Finally, following Sakellaridis and Venkatesh [16] we define a class of real spherical spaces which satisfy the wavefront lemma of Eskin-McMullen [7].

Acknowledgement: We thank the anonymous referee for useful suggestions which led to an improvement of our paper.

\section{REAL SPHERICAL SPACES}

2.1. Notation on real spherical spaces. We will denote Lie groups by upper case Latin letters, e.g $A, B$ etc., and their Lie algebras by lower case German letters, e.g. $\mathfrak{a}, \mathfrak{b}$ etc.

Let $G$ be an algebraic real reductive group by which we understand an open subgroup of the real points of a connected complex reductive algebraic group $G_{\mathbb{C}}$. Further we let $H<G$ be a closed subgroup such that there is a complex algebraic subgroup $H_{\mathbb{C}}<G_{\mathbb{C}}$ such that $G \cap H_{\mathbb{C}}=H$. Under these assumptions we refer to $Z=G / H$ as a real algebraic homogeneous space. We set $Z_{\mathbb{C}}=G_{\mathbb{C}} / H_{\mathbb{C}}$ and note that there is a natural $G$-equivariant embedding

$$
Z \hookrightarrow Z_{\mathbb{C}}, \quad g H \mapsto g H_{\mathbb{C}}
$$

Let us denote by $z_{0}=H$ the standard base point of $Z$. We denote by $\mathbb{C}\left[G_{\mathbb{C}}\right]$ the ring of regular functions on $G_{\mathbb{C}}$. 
Denote by $\mathcal{P}$ the variety of all minimal parabolics. Then $\mathcal{P} \simeq G / P$ for any given $P \in \mathcal{P}$. In this paper we will assume that $Z$ is real spherical, i.e., some, and hence all, $P \in \mathcal{P}$ admit an open orbit on $Z$.

2.2. Embeddings. It follows from a theorem of Chevalley that every real algebraic homogeneous space $Z=G / H$ admits a $G$-equivariant embedding into the projective space of a rational $G$-module $V$, i.e., there is a vector $0 \neq v_{H} \in V$ such that $H$ is the stabilizer of the line $\left[v_{H}\right] \in \mathbb{P}(V)$. Then the map

$$
Z=G / H \rightarrow \mathbb{P}(V), \quad g H \mapsto\left[\pi(g) v_{H}\right]
$$

is a $G$-equivariant embedding.

It can sometimes be useful to reduce matters to the quasi-affine situation of an embedding into $V$. This is achieved with the following standard trick. For every algebraic character $\chi: H \rightarrow \mathbb{R}^{\times}$we set

$$
G_{1}:=G \times \mathbb{R}^{\times}, \quad H_{1, \chi}:=\left\{\left(h, \chi^{-1}(h)\right) \in G_{1} \mid h \in H\right\},
$$

then $Z_{1, \chi}=G_{1} / H_{1, \chi}$ is a real spherical space. In particular, if $V$ and $v_{H}$ are as above, and if $\chi: H \rightarrow \mathbb{R}^{\times}$is given by $\pi(h) v_{H}=\chi(h) v_{H}$, then

$$
(g, t) H_{1, \chi} \mapsto t \pi(g) v_{H}, \quad Z_{1, \chi}:=G_{1} / H_{1, \chi} \rightarrow V
$$

is an embedding.

2.3. The local structure theorem. Let $P \in \mathcal{P}$ be such that $P H$ is open in $G$, that is $\mathfrak{g}=\mathfrak{p}+\mathfrak{h}$.

If $\mathfrak{l}$ is a real reductive Lie algebra then we denote by $\mathfrak{l}_{n}$, reps. $\mathfrak{l}_{c}$ the union of the non-compact, resp. compact, simple ideals of $\mathfrak{l}$. Note that

$$
\mathfrak{l}=\mathfrak{z}(\mathfrak{l}) \oplus \mathfrak{l}_{\mathrm{n}} \oplus \mathfrak{l}_{\mathrm{c}}
$$

is a direct sum of reductive Lie algebras.

According to the local structure theorem of [12, Thm. 2.2] there exists a parabolic subgroup $Q \supset P$ with Levi decomposition $Q=L U$ such that:

- $Q \cdot z_{0}=P \cdot z_{0}$ and

- $L_{\mathrm{n}}<Q \cap H<L$ with $L_{n}=\left\langle\exp \mathfrak{l}_{\mathrm{n}}\right\rangle$.

Hence on the level of Lie algebras we have

$$
\mathfrak{l}_{\mathrm{n}} \subset \mathfrak{q} \cap \mathfrak{h} \subset \mathfrak{l} .
$$

A parabolic subgroup $Q$ with the properties listed above is said to be $Z$-adapted. It is shown in [12, Thm. 2.7] that only one parabolic subgroup containing $P$ is $Z$-adapted .

We let $K_{L} A_{L} N_{L}=L$ be an Iwasawa decomposition of $L$ and set $A:=A_{L}$. This way, we obtain a Levi decomposition $P=M A N=$ $M A \ltimes N$ where $M=Z_{K_{L}}(A)$ and $N=N_{L} U$. 
We let

$$
\mathfrak{z}(\mathfrak{l})=\mathfrak{z}(\mathfrak{l})_{n p} \oplus \mathfrak{z}(\mathfrak{l})_{c p}
$$

be the decomposition into compact and non-compact part, i.e., $\mathfrak{z}(\mathfrak{l})_{c p}$ is the Lie algebra of the maximal compact subgroup of the abelian Lie group $Z(L)$ and $\mathfrak{z}(\mathfrak{l})_{n p}$ is its orthocomplement.

Let $\mathfrak{d}:=\mathfrak{z}(\mathfrak{l})+\mathfrak{l}_{c}$. As there is no algebraic homomorphism of a noncompact torus into a compact group we obtain that every algebraic subalgebra $\mathfrak{c}$ of $\mathfrak{d}$ decomposes $\mathfrak{c}=\left[\mathfrak{c} \cap \mathfrak{z}(\mathfrak{l})_{n p}\right] \oplus\left[\mathfrak{c} \cap\left(\mathfrak{z}(\mathfrak{l})_{c p}+\mathfrak{l}_{c}\right)\right]$. As $\mathfrak{h}$ is algebraic we thus get

$$
\mathfrak{h} \cap \mathfrak{l}=\mathfrak{l}_{\mathrm{n}}+\mathfrak{a}_{h}+\mathfrak{m}_{h}
$$

with $\mathfrak{a}_{h} \subset \mathfrak{z}(\mathfrak{l})_{n p}$ the image of $\mathfrak{h} \cap \mathfrak{l}$ under the orthogonal projection $\mathfrak{l} \rightarrow \mathfrak{z}(\mathfrak{l})_{n p}$ and likewise for $\mathfrak{m}_{h}<\mathfrak{z}(\mathfrak{l})_{c p}+\mathfrak{l}_{\mathrm{c}}$. Let $\mathfrak{a}_{Z} \subset \mathfrak{z}(\mathfrak{l})_{n p}$ be the orthogonal complement of $\mathfrak{a}_{h}$ and $\mathfrak{m}_{Z}$ the orthogonal complement to $\mathfrak{m}_{h}$ in $\mathfrak{l}_{c}+\mathfrak{z}(\mathfrak{l})_{c}$. Accordingly the following direct sum holds

$$
\mathfrak{g}=\mathfrak{h} \oplus \mathfrak{a}_{Z} \oplus \mathfrak{m}_{Z} \oplus \mathfrak{u} .
$$

2.4. The open $P$-orbits in $Z=G / H$. Let $P \in \mathcal{P}$ be such that $P H$ is open in $G$ and let $Q \supset P$ be $Z$-adapted. Our goal in this subsection is to describe all open $P$-orbits on $Z=G / H$.

Recall that the local structure theorem asserts that $P H=Q H$ and that there is an algebraic diffeomorphism

$$
U \times S \rightarrow P \cdot z_{0}
$$

where $S=L \cdot z_{0}$ is a homogeneous space for the group $D:=L / L_{\mathrm{n}}$. As $L$ is reductive we can and will assume that $D<L$. Note that the Lie algebra $\mathfrak{d}=\mathfrak{z}(\mathfrak{l})+\mathfrak{l}_{c}$ is compact and contained in $\mathfrak{a}+\mathfrak{m}$ (recall that a Lie algebra is called compact if it is isomorphic to the Lie algebra of a compact Lie group).

Lemma 2.1. $P_{\mathbb{C}} \cdot z_{0} \cap Z$ is the union of the open P-orbits in $Z$.

Proof. We first note that $P_{\mathbb{C}} \cdot z_{0}$ is Zariski open, hence dense and thus

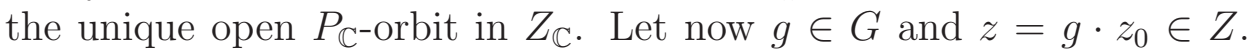
Then $\operatorname{Ad}(g)^{-1} \mathfrak{p}+\mathfrak{h}=\mathfrak{g}$ if and only if $\operatorname{Ad}(g)^{-1} \mathfrak{p}_{\mathbb{C}}+\mathfrak{h}_{\mathbb{C}}=\mathfrak{g}_{\mathbb{C}}$, and hence $P \cdot z$ is open in $Z$ if and only if $P_{\mathbb{C}} \cdot z$ is open in $Z_{\mathbb{C}}$. The lemma follows immediately.

The local structure theorem was obtained through the use of a $P$ semi-invariant regular function $f$ on $G$ (see also Section 3.4 where we review this in more detail). If we view $f$ as a regular function on $G_{\mathbb{C}}$ we obtain the complex version of the local structure theorem: with $Q_{\mathbb{C}}$ the 
Zariski closure of $Q$ and $U_{\mathbb{C}}:=\exp \left(\mathfrak{u}_{\mathbb{C}}\right)$ we obtain a parametrization of

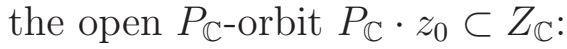

$$
U_{\mathbb{C}} \times S_{\mathbb{C}} \rightarrow P_{\mathbb{C}} \cdot z_{0} .
$$

The slice $S_{\mathbb{C}}$ is described as follows: With $L_{\mathbb{C}}<Q_{\mathbb{C}}$ the Levi part with $L_{\mathbb{C}} \supset L$ and $L_{n, \mathbb{C}}=\left\langle\exp \left(\mathfrak{l}_{n, \mathbb{C}}\right)\right\rangle$ we obtain that $S_{\mathbb{C}}=L_{\mathbb{C}} \cdot z_{0}$ is a homogeneous space for the group $D_{\mathbb{C}}:=L_{\mathbb{C}} / L_{n, \mathbb{C}}$.

Note that the number of open $P$-orbits in $Z$ is finite. In view of (2.6) we have $\left(P_{\mathbb{C}} \cdot z_{0}\right)(\mathbb{R})=U \times S_{\mathbb{C}}(\mathbb{R})$. Hence all open $P$-orbits are given by

$$
P t_{1} \cdot z_{0}, \ldots, P t_{m} \cdot z_{0}
$$

where $t_{j} \in \exp (i \mathfrak{d})$ (see also (2.10)). It is no loss of generality to assume that $t_{1}=1$. In particular we find $e_{j} \in G$ and $h_{j} \in H_{\mathbb{C}}$ such that $t_{j}=e_{j} h_{j}$. Set

$$
F:=\left\{e_{1}, \ldots, e_{m}\right\}
$$

and note that

$$
\mathrm{PgH} \subset G \text { is open for all } g \in F \text {. }
$$

2.5. Explicit structure of the slice $S$. As $D$ is an algebraic group we have

$$
D=Z(L)_{n p} \times D_{c}
$$

with $D_{c}$ a compact subgroup with Lie algebra $\mathfrak{z}(\mathfrak{l})_{c}+\mathfrak{l}_{c}$ and $Z(L)_{n p}:=$ $\exp \mathfrak{z}(\mathfrak{l})_{n p}$. With $C<D$ the stabilizer of $z_{0}$ in $D$ we obtain likewise that

$$
C=A_{h} \times M_{h}
$$

with $M_{h}$ a compact subgroup of $D_{c}$ with Lie algebra $\mathfrak{m}_{h}$. As $Z(L)_{n p}=$ $A_{Z} \times A_{h}$ we conclude that

$$
S=D / C \simeq A_{Z} \times D_{c} / M_{h}
$$

Set $M_{Z}:=D_{c} / M_{h}$. In particular, in (2.7) one can arrange that

$$
t_{j} \in T_{Z}:=\exp \left(i \mathfrak{a}_{Z}\right), \quad(j=1, \ldots, m) .
$$




\section{Simple COMPACTIFicAtions}

In the sequel we use the term $G$-space for a topological space endowed with a continuous $G$-action.

By a compactification of $Z=G / H$ we understand a compact $G$-space $\widehat{Z}$ such that

- $\widehat{Z} \supset Z$ as $G$-space.

- $Z$ is open dense in $\widehat{Z}$.

Compactifications of real spherical spaces exist: Recall from (2.1) the $G$-equivariant embedding in $\mathbb{P}(V)$.

According to [12] the closure $\widehat{Z}$ of $Z$ in $\mathbb{P}(V)$ has a finite orbit decomposition. However, the orbit structure of such an embedding can be quite complicated, in particular it can happen that there are closed $G$-orbits of different orbit type.

Example 3.1. We consider $G=\operatorname{SL}(2, \mathbb{R})$ with $H=N$. Then $G / H \simeq$ $\mathbb{R}^{2} \backslash\{0\}$ which we realize in $\mathbb{P}(V)$ where $V=\mathbb{R}^{2} \oplus \mathbb{R}$ via $v \mapsto[(v, 1)]$. The closure of $Z$ in $\mathbb{P}(V)$ consists of $Z$ and two closed orbits: The $G$ fixed point $[(0,1)]$ and the orbit $\mathbb{P}\left(\mathbb{R}^{2}\right) \simeq G / P$ through $\left[\left(v_{N}, 0\right)\right]$ where $v_{N} \in \mathbb{R}^{2}$ is $N$-fixed.

The goal of this section is to construct more suitable compactifications with a simple structure of the closed orbits.

Recall the parabolic $Q=L U \supset P$ which we attached to $Z$. In the sequel it is convenient to choose a Cartan involution $\theta$ on $G$ such that $L$ is $\theta$-stable. The opposite parabolic to $Q$ is then defined by $\bar{Q}:=\theta(Q)=L \bar{U}$ with $\bar{U}=\theta(U)$.

If $V$ is a finite dimensional real $G$-module, then we denote by $V^{*}$ its dual. We choose an inner product $\langle\cdot, \cdot\rangle$ on $V$ which is $\theta$-covariant, that is $\langle g \cdot v, w\rangle=\left\langle v, \theta(g)^{-1} \cdot w\right\rangle$ holds for all $g \in G$, and $v, w \in V$. We consider the linear identification

$$
V \rightarrow V^{*}, \quad v \mapsto v^{*}:=\langle\cdot, v\rangle
$$

and observe that the dual representation $V^{*}$ can be realized on $V$ but with the twisted $G$-action $g * v:=\theta(g) \cdot v$. In particular we see that the ray $\mathbb{R}^{+} v \subset V$ is stabilized by $\bar{Q}$ if and only if $\mathbb{R}^{+} v^{*} \subset V^{*}$ is stabilized by $Q$.

We call a compactification $\widehat{Z}$ simple provided that there exists only one closed $G$-orbit $Y \subset \widehat{Z}$ and $Y \simeq G / \bar{Q}$ as $G$-space.

Simple compactifications arise in the following context.

Lemma 3.2. Let $V$ be an irreducible finite dimensional real rational $G$-representation with the following properties: 
(1) There is a non-zero vector $v_{H} \in V$ such that the stabilizer of the line $\mathbb{R} v_{H}$ is $H$.

(2) There is a non-zero vector $v \in V$ for which the stabilizer of the ray $\mathbb{R}^{+} v$ is $\bar{Q}$.

Then the closure $\overline{G \cdot\left[v_{H}\right]} \subset \mathbb{P}(V)$ is a simple compactification of $Z=$ $G / H$ with closed orbit $Y=G \cdot[v]$.

Proof. The orbit $Y$ is isomorphic to $G / \bar{Q}$ by assumption. Since $\bar{Q}$ is a parabolic subgroup, $Y$ is compact and therefore closed in $\mathbb{P}(V)$. The action of $G$ on $\mathbb{P}(V)$ is algebraic, hence all orbits are locally closed. In particular, $\overline{G \cdot\left[v_{H}\right]}$ must contain a closed orbit. Thus it suffices to show that $Y$ is the only closed $G$-orbit of $\mathbb{P}(V)$. This is a standard fact whose proof, for the convenience to the reader, we briefly recall: Let $G \cdot[u]$ be a closed orbit and decompose $u$ in weight spaces for $\mathfrak{a}$. Since $V$ is irreducible we may assume $u$ has a non-trivial component in the lowest weight space $[v]$. A sequence $a_{n}$ of elements converging to infinity in the positive Weyl chamber $A^{+}$will now exhibit $[v]$ as the limit of $a_{n} \cdot[u]$.

3.1. The structure of $P_{\mathbb{C}} H_{\mathbb{C}}$. Let $P$ be a minimal parabolic subgroup such that $P H$ is open in $G$. Then $P_{\mathbb{C}} H_{\mathbb{C}}$ is open in $G_{\mathbb{C}}$ and we have:

Lemma 3.3. Let $H_{\mathbb{C}, 0}$ be the identity component of $H_{\mathbb{C}}$. Then

$$
P_{\mathbb{C}} H_{\mathbb{C}}=P_{\mathbb{C}} H_{\mathbb{C}, 0}
$$

Proof. This follows from the fact that $P_{\mathbb{C}} H_{\mathbb{C}, 0}$ is Zariski open in $G_{\mathbb{C}}$ and the fact that $G_{\mathbb{C}}$ is irreducible.

We denote by $\mathcal{P}_{+}^{\mathbb{C}}$ the multiplicative monoid of regular functions on $G_{\mathbb{C}}$ which have no zero in $P_{\mathbb{C}} H_{\mathbb{C}}$. Every $f \in \mathcal{P}_{+}^{\mathbb{C}}$ is of the form

$$
f(p h)=f(e) \chi(p) \psi(h) \quad p \in P_{\mathbb{C}}, h \in H_{\mathbb{C}, 0}
$$

with algebraic characters $\chi: P_{\mathbb{C}} \rightarrow \mathbb{C}^{*}$ and $\psi: H_{\mathbb{C}, 0} \rightarrow \mathbb{C}^{*}$. This follows from Rosenlicht's theorem, see [11] p. 78.

Lemma 3.4. The Zariski closed subset $G_{\mathbb{C}}-P_{\mathbb{C}} H_{\mathbb{C}}$ in $G_{\mathbb{C}}$ is affine and the zero locus of a regular function on $G_{\mathbb{C}}$.

Proof. Recall that a complex homogeneous space $D_{\mathbb{C}} / C_{\mathbb{C}}$ of a reductive group $D_{\mathbb{C}}$ by a reductive subgroup $C_{\mathbb{C}}$ is affine. Hence the local structure theorem (2.6) implies that $P_{\mathbb{C}} \cdot z_{0} \subset Z_{\mathbb{C}}$ is affine. It follows that the complement of $P_{\mathbb{C}} \cdot z_{0}$ is of pure codimension one in $Z_{\mathbb{C}}$ and likewise for $P_{\mathbb{C}} H_{\mathbb{C}} \subset G_{\mathbb{C}}$ (see [8], Prop. 1). 
It remains to show that the divisor $\mathcal{D}:=G_{\mathbb{C}}-P_{\mathbb{C}} H_{\mathbb{C}}$ is the zero locus of a regular function. This is a consequence of the fact that the Picard group of $G_{\mathbb{C}}$ is finite (cf. [11], Prop. 4.5).

Definition 3.5. We denote by $\mathcal{P}_{+} \subseteq \mathcal{P}_{+}^{\mathbb{C}}$ the set of regular functions $f$ on $G_{\mathbb{C}}$ for which

(1) $f(G) \subseteq \mathbb{R}$

(2) $f(m g)=f(g)$ for all $m \in M, g \in G$,

(3) $P_{\mathbb{C}} H_{\mathbb{C}} \subseteq\left\{g \in G_{\mathbb{C}} \mid f(g) \neq 0\right\}$.

and by $\mathcal{P}_{++} \subseteq \mathcal{P}_{+}$the subset with (3) replaced by

(4) $P_{\mathbb{C}} H_{\mathbb{C}}=\left\{g \in G_{\mathbb{C}} \mid f(g) \neq 0\right\}$.

Lemma 3.6. $\mathcal{P}_{++}$is not empty.

Proof. We obtain from Lemma 3.4 a function $f \in \mathbb{C}\left[G_{\mathbb{C}}\right]$ with $f(z)=0$ if and only if $z \notin P_{\mathbb{C}} H_{\mathbb{C}}$. For elements in $P_{\mathbb{C}} H_{\mathbb{C}}=P_{\mathbb{C}} H_{\mathbb{C}, 0}$ the identity (3.1) holds. Let $g \mapsto \bar{g}$ denote the complex conjugation of $G_{\mathbb{C}}$ with respect to $G$. Then the function $F(z)=f(z) \overline{f(\bar{z})}$ satisfies (11) and (4). As $M$ is compact, we observe that $|\chi(m)|^{2}=1$ for all $m \in M$. Hence $F$ satisfies (21) as well.

3.2. The left and right stabilizers of $P_{\mathbb{C}} H_{\mathbb{C}}$. In this subsection our concern is with the left and right stabilizers of the double coset $P_{\mathbb{C}} H_{\mathbb{C}}$ in $G_{\mathbb{C}}$. We denote by $L$ and $R$ the left and right regular representations of $G_{\mathbb{C}}$ on $\mathbb{C}\left[G_{\mathbb{C}}\right]$, that is,

$$
(L(g) f)(h)=f\left(g^{-1} h\right), \quad(R(g) f)(h)=f(h g),
$$

for $g, h \in G_{\mathbb{C}}$ and $f \in \mathbb{C}\left[G_{\mathbb{C}}\right]$.

We begin with the discussion of the left stabilizer.

Lemma 3.7. Let $H<G$ be a spherical subgroup and let $P$ and $Q$ be as above. Then

$$
Q_{\mathbb{C}}=\left\{g \in G_{\mathbb{C}} \mid g P_{\mathbb{C}} H_{\mathbb{C}}=P_{\mathbb{C}} H_{\mathbb{C}}\right\} .
$$

Furthermore, for $f \in \mathcal{P}_{++}$one has:

$$
Q=\left\{g \in G \mid L(g) f \in \mathbb{R}^{+} f\right\} .
$$

Proof. In [12, proof of Theorem 2.2, the parabolic subgroup $Q$ is obtained in an iterative procedure, by which a strictly decreasing sequence of real parabolic subgroups $Q_{0} \supset Q_{1} \supset \cdots \supset Q_{k}=Q$ is constructed. The parabolic subgroup $Q$ obtained in the final step is unique, and in particular independent of the initial subgroup $Q_{0}$. Following the proof in [12] it can be seen that in each step of the iteration, the set $\left(Q_{j+1}\right)_{\mathbb{C}} H_{\mathbb{C}}$ is a proper subset of the previous set $\left(Q_{j}\right)_{\mathbb{C}} H_{\mathbb{C}}$. In the 
first step of the iteration, as described in [12], this amounts to the fact that the function $F$ constructed there must satisfy $F(g)=0$ for some $g \in\left(G_{n}\right)_{\mathbb{C}}$, since it is a matrix coefficient for a non-trivial irreducible representation of this semisimple group.

We apply this procedure with $Q_{0}:=Q_{0, \mathbb{C}} \cap G$ where

$$
Q_{0, \mathbb{C}}=\left\{g \in G_{\mathbb{C}} \mid g P_{\mathbb{C}} H_{\mathbb{C}}=P_{\mathbb{C}} H_{\mathbb{C}}\right\} .
$$

Note that $Q_{0, \mathbb{C}}$ is a parabolic subgroup of $G_{\mathbb{C}}$ which is defined over $\mathbb{R}$, and hence $Q_{0}$ is a real parabolic subgroup with $\left(Q_{0}\right)_{\mathbb{C}}=Q_{0, \mathbb{C}}$. It follows from $Q H=P H$ that $Q \subseteq Q_{0}$. If $Q$ is strictly smaller than $Q_{0}$ the discussion above implies that $Q_{\mathbb{C}} H_{\mathbb{C}}$ is strictly smaller than $Q_{0, \mathbb{C}} H_{\mathbb{C}}$, which contradicts the definition of $Q_{0, \mathbb{C}}$. Hence $Q=Q_{0}$ and (3.2) is valid.

Finally let $f \in \mathcal{P}_{++}$and note that

$$
Q \subseteq\left\{g \in G \mid L(g) f \in \mathbb{R}^{+} f\right\} \subseteq Q_{0}
$$

by (3.1) and Definition 3.5. Hence (3.3) also follows.

Remark 3.8. The corresponding real version of (3.2), in which $P_{\mathbb{C}} H_{\mathbb{C}}$ is replaced by $P H$, is not true in general. For instance if $H=K$ is a maximal compact subgroup of $G$, then $P H=G$ and the left stabilizer in $G$ of $P H$ is $G$ whereas $P=Q$ in this case.

We move on to the discussion of the right stabilizer of $P_{\mathbb{C}} H_{\mathbb{C}}$ which we write as $J:=J_{\mathbb{C}} \cap G$ where

$$
J_{\mathbb{C}}:=\left\{g \in G_{\mathbb{C}} \mid P_{\mathbb{C}} H_{\mathbb{C}} g=P_{\mathbb{C}} H_{\mathbb{C}}\right\} .
$$

Furthermore, for $f \in \mathcal{P}_{++}$we set

$$
H_{f}^{\times}:=\left\{g \in G \mid R(g) f \in \mathbb{R}^{\times} f\right\} .
$$

Note that both $J$ and $H_{f}^{\times}$are closed algebraic subgroups of $G$

Lemma 3.9. One has

$$
H_{\mathbb{C}, 0} \cap G \subset H_{f}^{\times} \subset J
$$

and

$$
H \subset N_{G}(H) \subset J .
$$

In particular, $H_{f}^{\times}, N_{G}(H)$, and $J$ are spherical subgroups.

Proof. The inclusions in (3.6) follow from (3.1) and from Condition (44) of Definition 3.5, respectively.

The normalizer of $H$ acts on the set of open $H_{\mathbb{C}^{- \text {orbits }}}$ in $P_{\mathbb{C}} \backslash G_{\mathbb{C}}$. Since there is only one, (3.7) follows. 
Example 3.10. It can happen that $J / N_{G}(H)$ is of positive dimension. For that let $G=\mathrm{SO}(1, n)$ with $n \geq 3$ and let $P=M A N$ be a minimal parabolic subgroup of $G$. Then $M=\mathrm{SO}(n-1)$. Let $M^{\prime}<M$ be any subgroup which is transitive on the $n-2$-sphere. For instance if $n-1=2 k$, then $M^{\prime}=U(k)$ is such a group. Further, $H=M^{\prime} A$ is a self-normalizing spherical subgroup with $J=M A$. (see [14] for all that).

For a topological group $G$ we denote by $G_{0}$ its identity component.

Lemma 3.11. The following assertions hold:

(a) For $f \in \mathcal{P}_{++}$one has

$$
\left(H_{f}^{\times}\right)_{0}=J_{0} .
$$

In particular $\mathfrak{h}_{f}^{\times}=\mathfrak{j}$ is independent of the choice of $f \in \mathcal{P}_{++}$.

(b) There exists $f \in \mathcal{P}_{++}$such that

$$
H_{f}^{\times}=J \text {. }
$$

Proof. (a) The inclusion $\subseteq$ is obvious from (3.6). The function $f$ is non-zero, hence invertible on $P_{\mathbb{C}} H_{\mathbb{C}} \supset J_{\mathbb{C}}$. Any invertible function is an eigenfunction for a connected group (see e.g [11] Prop. 1.3). Hence $J_{0} \subseteq H_{f}^{\times}$.

As for (b) note that $J_{0}=\left(H_{f}^{\times}\right)_{0}$. Let $g_{1}, \ldots, g_{k} \in J$ be elements such that $J=\bigcup J_{0} g_{j}$, with a disjoint union. Let $f \in \mathcal{P}_{++}$. Then $F:=\prod_{j=1}^{k} R\left(g_{j}\right) f$ lies in $\mathcal{P}_{++}$and has the desired property.

Lemma 3.12. We have

$$
\mathfrak{h} \subset \mathfrak{j} \subset \mathfrak{h}+\mathfrak{d}
$$

Proof. By Lemma 3.11 it suffices to show for $f \in \mathcal{P}_{++}$that

$$
\mathfrak{h}_{f}^{\times} \subset \mathfrak{h}+\mathfrak{d} .
$$

Let $\phi: Z=G / H \rightarrow Z_{f}:=G / H_{f}^{\times}$be the canonical map. Let $Q_{f} \supseteq P$ the unique $Z_{f}$-adapted parabolic above $P$ (see Section 2.3). It follows from the construction of the adapted parabolics (which we just summarized in the proof of Lemma 3.7) that $Q=Q_{f}$. Now we apply the local structure theorem to $Z$ and $Z_{f}$. Let $S \subseteq Z$ and $S_{f} \subseteq Z_{f}$ be slices. Since they are constructed from the same $f$, we have $S=\phi^{-1}\left(S_{f}\right)$. Since $S$ is homogeneous for $D$, this implies the assertion $\mathfrak{d}+\mathfrak{h}=\mathfrak{d}+\mathfrak{h}_{f}^{\times}$.

Recall the notion of real rank of $Z$ from [12] which is an invariant of the real spherical space $Z$ and given by $\operatorname{rank}_{\mathbb{R}} Z=\operatorname{dim} \mathfrak{a}_{Z}$.

Note that if $Z$ is quasi-affine, then the complement of the open $P_{\mathbb{C}^{-}}$ orbit in $Z_{\mathbb{C}}$ is the zero locus of some $P$-semiinvariant regular function on 
$Z$. Its pull-back $f$ to $G$ is therefore in $\mathcal{P}_{++}$with trivial right character $\psi$. We denote by $\mathcal{P}_{++, 1}$ the subset of $\mathcal{P}_{++}$which corresponds to $\psi=\mathbf{1}$ on $H_{\mathbb{C}, 0}$, and set for this case

$$
I:=\bigcap_{f \in \mathcal{P}_{++, 1}} H_{f}, \quad H_{f}:=\{g \in G \mid R(g) f=f\} .
$$

Then $H_{\mathbb{C}, 0} \cap G \subset I$ and

$$
I \subset H_{f} \subset J .
$$

We obtain a refinement of Lemma 3.12 as follows.

Proposition 3.13. Suppose that $Z$ is quasi-affine. Then the following assertions hold:

(1) $\operatorname{rank}_{\mathbb{R}} G / H=\operatorname{rank}_{\mathbb{R}} G / I$.

(2) $\mathfrak{i} \subseteq \mathfrak{h}+\mathfrak{d}_{c}$.

(3) $\mathfrak{j} \subseteq \mathfrak{i}+\mathfrak{a}_{Z}$.

Proof. By comparing $P$-semiinvariants of $G / H$ on the slice, the local structure theorem implies that $\operatorname{rank}_{\mathbb{R}} G / H$ is also the rank of the lattice spanned by $P_{++, \mathbf{1}}$. Since by definition these are the semiinvariants of $G / I$, this shows the first assertion. Moreover, the equality of ranks shows that the map between the slices of $G / H_{0}$ and $G / I_{0}$ has compact fibers. This implies the second assertion. The last assertion follows from the fact that $\mathfrak{h}_{f}^{\times} \subseteq \mathfrak{h}_{f}+\mathfrak{a}_{Z}$ for all $f \in \mathcal{P}_{++, 1}$.

If $G$ is a real algebraic group, then we denote by $G_{\mathrm{n}}$ the normal subgroup generated by all unipotent elements. Note that $G_{\mathrm{n}}$ is connected, and that if $G=L \ltimes R_{u}$ is an arbitrary Levi decomposition, then $G_{n}=L_{n} \ltimes R_{u}$, where $L_{n}$ is as defined in Section 2.3. In particular, $G / G_{\mathrm{n}}$ has compact Lie algebra.

In the sequel we view the elements of $\mathbb{C}\left[Z_{\mathbb{C}}\right]$ as right $H_{\mathbb{C} \text {-invariant }}$ regular functions on $G_{\mathbb{C}}$.

Lemma 3.14. The space $\mathbb{C}\left[Z_{\mathbb{C}}\right]=\mathbb{C}\left[G_{\mathbb{C}}\right]^{H_{\mathbb{C}}}$ of right $H_{\mathbb{C}}$-invariant regular functions on $G_{\mathbb{C}}$ is right $J_{\mathrm{n}, \mathbb{C}}$-invariant.

Proof. Let $B_{\mathbb{C}} \subset P_{\mathbb{C}}$ be a Borel subgroup. To prove the lemma it is sufficient to show that every left $B_{\mathbb{C}^{-}}$eigenspace in $\mathbb{C}\left[Z_{\mathbb{C}}\right]$ is fixed by $J_{\mathrm{n}, \mathbb{C}}$ under the right regular action. Let $f$ be such an eigenfunction. Suppose first that $f$ is in fact a $P_{\mathbb{C}^{-}}$-eigenfunction, i.e., $f \in \mathcal{P}_{+}^{\mathbb{C}}$ is attached to a pair of characters $(\chi, \psi)$ on $P_{\mathbb{C}} H_{\mathbb{C}}=P_{\mathbb{C}} H_{\mathbb{C}, 0}$. As in the proof of Lemma 3.11(a) we obtain that $R(n) f$ is a multiple of $f$ for all $n \in\left(J_{\mathbb{C}}\right)_{0}$.

In general a $B_{\mathbb{C}^{-}}$-igenfunction does not need to be a $P_{\mathbb{C}^{-}}$-eigenfunction. To overcome this difficulty we use the method of $M$-averages of [12]: 
Let $\mathbb{C}[G]$ denote the ring of regular functions on $G$, i.e., the restrictions to $G$ of functions in $\mathbb{C}\left[G_{\mathbb{C}}\right]$. Using the $M$-average

$$
F \mapsto F^{M}(g)=\int_{M} F(m g) d m, \quad(g \in G)
$$

(with normalized Haar measure $d m$ on the compact group $M$ ), we obtain a quadratic map

$$
\mathbb{C}\left[G_{\mathbb{C}}\right] \rightarrow \mathbb{C}[G]^{M}, \quad \phi \mapsto \tilde{\phi} ; \quad \tilde{\phi}:=\left(\left.|\phi|_{G}\right|^{2}\right)^{M} .
$$

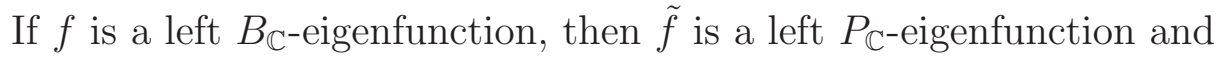
by what we just established we conclude that

$$
R(n) \tilde{f}=\tilde{f} \quad\left(n \in J_{\mathrm{n}, \mathbb{C}}\right) .
$$

Further observe that by right equivariance of $\phi \mapsto \tilde{\phi}$

$$
[R(n) f]^{\sim}=R(n) \tilde{f}=\tilde{f} \quad\left(n \in J_{\mathrm{n}, \mathbb{C}}\right) .
$$

Let $u(t), t \in \mathbb{R}$, be a unipotent one parameter subgroup of $J_{\mathrm{n}}$. Note that $V:=\operatorname{span}\{R(u(t)) f \mid t \in \mathbb{R}\}$ is a finite dimensional subspace. We find elements $F_{0}, \ldots, F_{N}$ of $V$ with $F_{0}=f$ and $F_{N} \neq 0$ such that

$$
R(u(t)) f=F_{0}+t F_{1}+\ldots+t^{N} F_{N}, \quad(t \in \mathbb{R}) .
$$

When applying the quadratic map from above we obtain

$$
[R(u(t)) f]^{\sim}=t^{2 N} \widetilde{F_{N}}+\text { lower order terms in } t .
$$

From $F_{N} \neq 0$ it follows that $\widetilde{F_{N}} \neq 0$, and from (3.10) we then deduce $N=0$. The lemma is proved.

Corollary 3.15. $H_{\mathrm{n}}=J_{\mathrm{n}}$.

Proof. We may assume that $Z$ is quasiaffine. First it is clear that $H_{\mathrm{n}} \subseteq J_{\mathrm{n}}$. To obtain the converse, let us denote by $\operatorname{Aut}\left(Z_{\mathbb{C}}\right)$ the group of birational automorphisms of $Z_{\mathbb{C}}$ and by $\operatorname{Aut}_{G_{\mathbb{C}}}\left(Z_{\mathbb{C}}\right)$ the subgroup of $G_{\mathbb{C}^{-}}$equivariant ones therein. Note that $N_{G_{\mathbb{C}}}\left(H_{\mathbb{C}}\right)$ naturally identifies with $\operatorname{Aut}_{G_{\mathbb{C}}}\left(Z_{\mathbb{C}}\right)$. The group $\operatorname{Aut}\left(Z_{\mathbb{C}}\right)$ is isomorphic to the automorphism group of the function field $\mathbb{C}\left(Z_{\mathbb{C}}\right)$. The fact that $Z$ and hence $Z_{\mathbb{C}}$ are quasiaffine implies that $\mathbb{C}\left(Z_{\mathbb{C}}\right)$ is the quotient field of $\mathbb{C}\left[Z_{\mathbb{C}}\right]$. According to Lemma 3.14 the group $J_{n, \mathbb{C}}$ preserves $\mathbb{C}\left[Z_{\mathbb{C}}\right]$ and hence $J_{\mathrm{n}, \mathbb{C}} \subseteq N_{G_{\mathbb{C}}}\left(H_{\mathbb{C}}\right)$. As $N_{G_{\mathbb{C}}}\left(H_{\mathbb{C}}\right) / H_{\mathbb{C}}$ has no real unipotent elements (12] Cor. 4.3), the corollary follows.

Recall the finite set $F$ from (2.8). The following result, which is analogous to [5], Prop. 5.1, implies the first assertion of Theorem 1.2. 
Theorem 3.16. Assume $N_{G}(H)=H$. Then there exists a compact subgroup $M_{J}<J$ with Lie algebra $\mathfrak{m}_{J} \subseteq \mathfrak{d}_{c}$ and

$$
J=M_{J} H \text {. }
$$

Furthermore one has

$$
J \subseteq(M \cap J) F H
$$

Proof. The group $J / J_{n}$ is compact modulo its center, and thus $J=$ $M_{J} Z_{1}$ where $M_{J}$ is compact and where $Z_{1}=\left\{g \in J \mid g J_{n} \in Z\left(G / J_{n}\right)\right\}$. Further, Proposition 3.13(2)-(3) implies that $\mathfrak{m}_{J} \subseteq \mathfrak{d}_{c}$.

Note that if $g J_{n} \in Z\left(J / J_{n}\right)$ then $g \in N_{G}(H)$ since $J_{n} \subset H \subset J$ by Corollary 3.15. Since $H=N_{G}(H)$ we conclude that (3.11) is valid.

We have $J_{\mathbb{C}} \subset Q_{\mathbb{C}} H_{\mathbb{C}}$ and hence

$$
J_{\mathbb{C}} \cdot z_{0}=\left(Q_{\mathbb{C}} \cap J_{\mathbb{C}}\right) \cdot z_{0}=\left(L_{\mathbb{C}} \cap J_{\mathbb{C}}\right) \cdot z_{0} \cdot
$$

As $L_{n, \mathbb{C}} \subset H_{\mathbb{C}}$ and $M_{\mathbb{C}} A_{\mathbb{C}} \rightarrow L_{\mathbb{C}} / L_{n, \mathbb{C}}$ is onto we conclude that

$$
J_{\mathbb{C}} \cdot z_{0}=\left(M_{\mathbb{C}} A_{\mathbb{C}} \cap J_{\mathbb{C}}\right) \cdot z_{0} .
$$

The last assertion of the theorem now follows by taking real points.

3.3. The existence of simple compactifications. We are now ready to prove the second assertion of Theorem [1.2, By Lemma 3.11 there exists $f \in \mathcal{P}_{++}$such that $H_{f}^{\times}=J$.

Theorem 3.17. Let $Z=G / H$ be a real spherical space. Let $f \in \mathcal{P}_{++}$ such that $H_{f}^{\times}=J$ and let $V_{f}:=\operatorname{span}_{\mathbb{R}}\{R(g) f \mid g \in G\}$ be the real representation of $G$ generated by $f$. Then

$$
Z_{J}:=G / J \rightarrow \mathbb{P}\left(V_{f}\right), \quad g H \mapsto R(g) f
$$

is a G-equivariant embedding. The closure $\widehat{Z}_{f}$ of the image provides a simple compactification of $Z_{J}$.

Proof. As $J=H_{f}^{\times}$, the map (3.12) determines a $G$-equivariant embedding from $Z_{J}$ into $\mathbb{P}\left(V_{f}\right)$. Note that $V_{f}$ is irreducible and that $f$ can be written as a matrix coefficient, that is $f(g)=v_{0}^{*}\left(g \cdot v_{H}\right)$ for some $J$-eigenvector $v_{H} \in V=V_{f}$ and a $Q$-eigenvector $v_{0}^{*}$ in the dual $V^{*}$. More precisely, $v_{H}$ is $f$ and $v_{0}^{*}$ is the evaluation at 1 . It follows from Lemma 3.7 that $Q$ is the stabilizer of $\mathbb{R}^{+} v_{0}^{*}$. With Lemma 3.2 we thus obtain the result. 
3.4. Coordinates near the closed orbit. In this section we assume that $H=J$. Further we let $\widehat{Z}=\widehat{Z}_{f}$ be a simple compactification constructed out of a function $f \in \mathcal{P}_{++}$as in Theorem 3.17. Let $Y$ be the unique closed orbit in $\widehat{Z}$. We recall that if $Q=L U$ is a Levi decomposition of the type discussed in Sections 2.3 2.4, we have defined $D:=L / L_{n}$ with compact Lie algebra $\mathfrak{d}=\mathfrak{z}(\mathfrak{l})+\mathfrak{l}_{c}$.

Proposition 3.18. There is an affine $Q$-invariant open subset $\widehat{Z}_{0} \subset \widehat{Z}$, a Levi decomposition $Q=L U$ as mentioned above, and an affine $L$ invariant subvariety $S_{Y}$ of $\widehat{Z}_{0}$ such that:

(1) The decomposition (2.5) is valid with $S=L \cdot z_{0} \subset S_{Y}$.

(2) $U \times S_{Y} \rightarrow \widehat{Z}_{0}, \quad(u, s) \mapsto u \cdot s$ is a homeomorphism.

(3) $S_{Y}$ is pointwise fixed by $L_{\mathrm{n}}$ and decomposes into finitely many D-orbits.

(4) $\widehat{Z}_{0} \cap Z$ is the union of the open P-orbits in $Z$, and $S_{Y} \cap Z$ consists of the open D-orbits in $S_{Y}$.

(5) $S_{Y} \cap Y=\{\widehat{z}\}$ where $\widehat{z}$ is the origin of $Y=G / \bar{Q}$.

Proof. In [12, Section 3 and in particular Th. 3.11, we derived a local structure theorem for real algebraic varieties which applies to $\widehat{Z}$. The construction was explicit and based on finite dimensional representation theory. We use the finite dimensional representation $V:=V_{f}$ with $H$-semi-spherical vector $f:=v_{H}$. Further we pick the $Q$-eigenvector $v_{0}^{*}$ in the dual of $V^{*}$ of $V$. With the data $\left(V, v_{H}, v_{0}^{*}\right)$ we begin the construction of the local slices as in [12, Section 3. One obtains an affine open set $\widehat{Z}_{0} \subset \widehat{Z}$ by

$$
\widehat{Z}_{0}=\widehat{Z} \cap\left\{[v] \in \mathbb{P}(V) \mid v_{0}^{*}(v) \neq 0\right\}
$$

and a $Q$-equivariant moment type map

$$
\mu: \widehat{Z}_{0} \rightarrow \mathfrak{g}^{*} ; \mu([v])(X):=\frac{v_{0}^{*}(X \cdot v)}{v_{0}^{*}(v)} .
$$

A Levi decomposition $Q=L U$ that leads to (2.5) is then obtained with $L$ being the stabilizer in of $\mu\left(z_{0}\right)=\mu\left(\left[v_{H}\right]\right) \in \mathfrak{g}^{*}$ in the coadjoint representation of $Q$, and with $S:=\mu^{-1}\left\{\mu\left(z_{0}\right)\right\} \cap P \cdot z_{0}$. It follows from (2.5) that $S=L \cdot z_{0}$, and (1) is established except for the final inclusion.

With these choices we let $\bar{Q}$ be the parabolic subgroup opposite to $Q$. Let $\widehat{z}=\left[v_{0}\right]$ for the (up to scalar) unique $\bar{Q}$-eigenvector $v_{0} \in V$. Note that $v_{0}^{*}\left(v_{0}\right) \neq 0$ and thus $\widehat{z} \in \widehat{Z}_{0}$. The slice $S_{Y}$ is defined by $\mu^{-1}\{\mu(\widehat{z})\}$. In particular, since $\widehat{z}$ is fixed by $L$, the stabilizer in $Q$ of $\mu(\widehat{z}) \in \mathfrak{g}^{*}$ is a Levi subgroup that contains and hence equals $L$. Note that we do not need any iterations of the construction as in [12] as $Q$ 
is already the $Z$-adapted parabolic. The assertions in (2) and (3) now follow from [12], Th. 3.10 and 3.11.

It follows from (2) that there exists unique elements $u \in U$ and $s \in S_{Y}$ such that $z_{0}=u \cdot s$. Then $\mu\left(z_{0}\right)=\operatorname{Ad}^{*}(u) \mu(s)=\operatorname{Ad}^{*}(u) \mu(\widehat{z})$ and hence $\mu\left(z_{0}\right)$ is stabilized by $u L u^{-1}$. Hence $L=u L u^{-1}$ and since $U$ acts freely on the set of Levi subgroups in $Q$ we conclude $u=\mathbf{1}$. Then $z_{0}=s \in S_{Y}$ and the final inclusion of (11) follows.

The first assertion in (44) follows from the fact that $f \in \mathcal{P}_{++}$. Indeed the construction in 12 yields that $\widehat{Z}_{0} \cap Z$ coincides with the nonvanishing locus of $f$, i.e., the union of all open $P$-orbits in $Z$ (see Lemma 2.1). Because of (2)-(3), the second assertion of (4) is an immediate consequence of the first.

For (5) we remark first that $\widehat{z}$ belongs to $S_{Y}$ and is $\bar{Q}$-fixed, in particular $D$-fixed. It remains to show there are no other elements from $Y$ in $S_{Y}$. Let $\widehat{y}=\left[g \cdot v_{0}\right] \in Y$ for some $g \in G$. As $G=U \mathcal{W}_{A} \bar{Q}$ for the Weyl group $\mathcal{W}_{A}$ attached to $A$, we we may assume that $g=u w$ for some $w \in \mathcal{W}_{A}$ and $u \in U$. If also $\widehat{y} \in S_{Y}$, then $v_{0}^{*}\left(g \cdot v_{0}\right) \neq 0$. Hence we may assume $w=\mathbf{1}$ as $v_{0}^{*}\left(u w \cdot v_{0}\right)=v_{0}^{*}\left(w \cdot v_{0}\right)$, which is zero if $w \cdot v_{0} \neq v_{0}$. We claim that $u=\mathbf{1}$.

Assume $u \neq \mathbf{1}$ and set $u=\exp (Y)$ for $Y \in \mathfrak{u}$. Now $\widehat{y} \in S_{Y}$ implies $\mu(\widehat{y})=\mu(\widehat{z})$ and so

$$
v_{0}^{*}\left(X \cdot v_{0}\right)=v_{0}^{*}\left(X \cdot u \cdot v_{0}\right)
$$

for all $X \in \mathfrak{g}$ by (3.13). For $X \in \mathfrak{u}$ the left hand side of (3.14) vanishes, whereas the other side is

$$
v_{0}^{*}\left(\operatorname{Ad}\left(u^{-1}\right) X \cdot v_{0}\right)=v_{0}^{*}\left(e^{-\operatorname{ad} Y} X \cdot v_{0}\right) .
$$

Let $p: \mathfrak{g} \rightarrow \mathfrak{z}(\mathfrak{l})$ be the projection along $\mathfrak{u}+\overline{\mathfrak{u}}+[\mathfrak{l}, \mathfrak{l}]$ and observe that

$$
v_{0}^{*}\left(e^{-\operatorname{ad} Y} X \cdot v_{0}\right)=\lambda\left(p\left(e^{-\operatorname{ad} Y} X\right)\right) v_{0}^{*}\left(v_{0}\right)
$$

where $\lambda \in \mathfrak{a}^{*}$ is the $\mathfrak{a}$-weight of $v_{0}$. Hence $\lambda\left(p\left(e^{-\operatorname{ad} Y} X\right)\right)=0$. Write $Y=\sum Y_{\alpha}$ as a sum of $\mathfrak{a}$-weight vectors and let $\beta$ be the smallest root for which $Y_{\beta} \neq 0$. Then for $X=\theta\left(Y_{\beta}\right)$ we obtain

$$
p\left(e^{-\operatorname{ad} Y} X\right)=-\left[Y_{\beta}, \theta\left(Y_{\beta}\right)\right] .
$$

As $\bar{Q}$ is the stabilizer of $\left[v_{0}\right]$, the weight $\lambda$ is non-zero on $\left[Y_{\beta}, \theta\left(Y_{\beta}\right)\right]$ for every root $\beta$ of $\mathfrak{u}$. We reached a contradiction, hence $u=\mathbf{1}$ and (5) holds.

Later in the text we will use Proposition 3.18 in a slightly disguised form which we explicate in the following corollary. 
As in (2.7) let $t_{1}, \ldots, t_{m} \in T_{Z}$ parametrize the open $P$-orbits on $Z$ and let $S_{Z, j}$ be the closure of $S_{Z, j}^{\prime}=D t_{j} \cdot z_{0}$ in $\widehat{Z}_{0}$. We set

$$
S_{Z}:=\bigcup_{j=1}^{m} S_{Z, j} \quad \text { and } \quad S_{Z}^{\prime}:=\bigcup_{j=1}^{m} S_{Z, j}^{\prime} .
$$

Corollary 3.19. Within the notation of Proposition [3.18, $S_{Z}=S_{Y}$. In particular the following assertions hold:

(1) $\widehat{Z}_{0}=U S_{Z}$.

(2) $S_{Z} \cap Z=S_{Z}^{\prime}$

(3) $S_{Z} \cap Y=\{\widehat{z}\}$.

Proof. Since $z_{0} \in S_{Y}$ we find $S_{Z, 1} \subset S_{Y}$. By Proposition 3.18 there exist for each $j$ unique elements $u_{j} \in U$ and $s_{j} \in S_{Y}$ such that $t_{j} \cdot z_{0}=u_{j} \cdot s_{j}$. Then $s_{j} \in S_{Y, j}^{\prime}:=S_{Y} \cap\left(P t_{j} \cdot z_{0}\right)$ and hence the $D$-orbits $u_{j} \cdot S_{Y, j}^{\prime}$ and $S_{Z, j}^{\prime}$ agree. By (2.6) the elements $u_{j} \in U$ all have to be equal to $u_{1}=1$. Hence $S_{Z}=S_{Y}$.

\section{Polar decomposition}

We recall that for a minimal parabolic subgroup $P$ with $P H$ open, we chose a specific Cartan involution $\theta$ of $G$, which was adapted to the geometry of $P H$. The corresponding Langlands decomposition of $P$ is denoted $P=M A N$, and the corresponding maximal compact subgroup of $G$ is denoted by $K$. Then $M \subset K$. Recall the finite set $F$ from (2.8) and its property (2.9).

Lemma 4.1. Suppose that $Z=G / H$ is a homogeneous real spherical space and $P$ a minimal parabolic subgroup of $G$ such that $P H$ is open. Then there exist finite sets $F^{\prime}, F^{\prime \prime} \subset G$ such that

$$
G=F^{\prime} K A_{Z} F^{\prime \prime} H \text {. }
$$

Moreover $\mathrm{PgH}$ is open for all $g \in F^{\prime \prime}$, and in case $H=N_{G}(H)$ one has $F^{\prime \prime}=F$.

Proof. Recall from [12] Prop. 4.2 that $N_{G}(\mathfrak{h})_{0} \subset M A_{Z} H$. Hence it suffices to show

$$
G=F^{\prime} K A_{Z} F N_{G}(\mathfrak{h})_{0}
$$

and we may assume that $\mathfrak{h}$ is self-normalizing. It then follows that $N_{G}\left(N_{G}(\mathfrak{h})\right)=N_{G}(\mathfrak{h})$ and hence we can apply Theorem 3.16 to the spherical subgroup $N_{G}(\mathfrak{h})$. In this context observe also that $\mathrm{PgH}$ is open for all $g \in M A F N_{G}(\mathfrak{h})$. By that we can then reduce all statements to the case where $H=J$, which we assume henceforth.

We let $\widehat{Z}=\widehat{Z}_{f}$ be a simple compactification as constructed before. 
Let $Y \subset \widehat{Z}$ be the the unique closed boundary orbit. We use Proposition 3.18 and its Corollary 3.19 to obtain a $P$-stable affine open set $\widehat{Z}_{0}=U S_{Z} \subset \widehat{Z}$ with the properties (1)-(3) listed in Corollary 3.19.

Consider the map

$$
\Phi: K \times S_{Z} \rightarrow \widehat{Z}, \quad(k, s) \mapsto k \cdot s .
$$

Lemma 4.2. The map $\Phi$ is open at $\widehat{z}$.

Proof. This is easily seen if $\widehat{z}$ is a smooth point of the affine variety $S_{Z}$. Indeed, as the projection $\mathfrak{k} \rightarrow \mathfrak{u} \simeq \mathfrak{g} / \overline{\mathfrak{q}}$ is surjective, we see that the differential $d \Phi$ is surjective at $(\mathbf{1}, \widehat{z})$.

We now consider the general case. As in [4], Sect. 1.2, the slice is induced from a linear slice in the surrounding projective space $\mathbb{P}\left(V_{f}\right)$ of $\widehat{Z}$ : Let $\mathbb{P}\left(V_{f}\right)_{0}:=\left\{[v] \in \mathbb{P}\left(V_{f}\right) \mid v_{0}^{*}(v) \neq 0\right\}$ and note that $\mathbb{P}\left(V_{f}\right)_{0} \simeq$ $v_{0}+\operatorname{ker} v_{0}^{*}$ is affine open. In analogy to Prop. 1.2 in [4] we have that

$$
\Psi: U \times\left(\mathbb{R}^{\times} v_{0}+\left(\mathfrak{g} \cdot v_{0}^{*}\right)^{\perp}\right) \rightarrow \mathbb{P}\left(V_{f}\right)_{0}, \quad(n, w) \mapsto[n \cdot w]
$$

is a diffeomorphism. The slice $S_{Z}$ is obtained from the intersection of $\widehat{Z}$ with $\mathbb{R}^{\times}\left(v_{0}+\left(\mathfrak{g} \cdot v_{0}^{*}\right)^{\perp}\right)$. As $K \cdot \widehat{z}=G / \bar{Q}$, it follows that the differentiable map

$$
\Psi^{\prime}: K \times\left(\mathbb{R}^{\times} v_{0}+\left(\mathfrak{g} \cdot v_{0}^{*}\right)^{\perp}\right) \rightarrow \mathbb{P}\left(V_{f}\right)_{0}, \quad(k, w) \mapsto[k \cdot w]
$$

has surjective differential at $\left(\mathbf{1}, v_{0}\right) \leftrightarrow \widehat{z}$. In particular we can conclude that $\Phi$ is an open map near $\widehat{z}$.

We can now complete the proof of Lemma 4.1. As $\widehat{z}$ is $D_{c}$-fixed we find a $D_{c}$-invariant open neighborhood $S_{Z}^{1} \subset S_{Z}$ containing $\widehat{z}$ such that $K \cdot S_{Z}^{1}=: \mathcal{U}$ contains an open neighborhood of $Y$ in $\widehat{Z}$.

We may assume that $D_{c} \subset K$ and thus replace $S_{Z}$ by $S_{Z} / D_{c}$ which is a finite union of $A_{Z}$-orbits (the real points of a toric variety). These finitely many $A_{Z}$-orbits we realize in $S_{Z}$ as the set $S_{Z}^{2}$, i.e., we choose a continuous embedding $S_{Z} / D_{c} \hookrightarrow S_{Z}$ and let $S_{Z}^{2}$ be the image. The piece in $S_{Z}^{2}$ which corresponds to $S_{Z}^{1}$ we denote by $S_{Z}^{3}$. Let $S_{Z}^{3 \prime}:=S_{Z}^{3} \cap Z$ and recall from Corollary 3.19 that

$$
S_{Z}^{3^{\prime}} \subset D F \cdot z_{0}=D_{c} A_{Z} F \cdot z_{0} .
$$

We find for every element $z \in \widehat{Z}$ an element $g_{z} \in G$ such that $g_{z}^{-1} \cdot z \in S_{Z, 3}$. As $\widehat{Z}$ is compact, we conclude that finitely many $g_{z}$, say $g_{1}, \ldots, g_{N}$ suffice so that $\bigcup_{j=1}^{N} g_{j} K S_{Z, 3}=\widehat{Z}$. We obtain with (4.1) the assertion of the lemma.

We reformulate Lemma 4.1 in a more compact form and obtain: 
Theorem 4.3. (Polar decomposition) Suppose that $Z=G / H$ is a homogeneous real spherical space and $P$ be a minimal parabolic subgroup of $G$ such that $P H$ is open. Then there exists a compact subset $\Omega \subset G$ and a finite set $F^{\prime \prime} \subset G$ such that

$$
G=\Omega A_{Z} F^{\prime \prime} H
$$

Moreover $\mathrm{PgH}$ is open for all $g \in F^{\prime \prime}$.

In particular, Theorem 1.1 follows.

\section{Compression CONES AND THE Fine CONVEX GEOMETRY NEAR THE CLOSED ORBIT}

In this section we will investigate the fine convex geometry of the slice $S_{Z}$ near the $\bar{Q}$-fixed point $\widehat{z}$.

5.1. Compression cones of $H$-spherical representations. We begin with a remark about finite dimensional representations. The CartanHelgason theorem asserts that an irreducible finite dimensional representation of $G$ has an $M N$-invariant highest weight vector if and only if it is $K$-spherical. All finite dimensional irreducible representations considered in this section are assumed to be of this type. It is known that these representations admit a real structure and are self-dual.

Let $(\pi, V)$ be finite dimensional real irreducible representation which is $H$-semi-spherical, i.e. there exists a non-zero vector $v_{H} \in V$ and an algebraic character $\chi: H \rightarrow \mathbb{R}^{\times}$such that $H$ acts on $v_{H}$ by $\chi$. By the assumption above there exists a highest weight $\lambda \in \mathfrak{a}^{*}$ of the dual representation $\left(\pi^{*}, V^{*}\right)$ and a highest weight vector $v_{\lambda}^{*} \in V^{*}$ (unique up to scaling) with

$$
(\operatorname{man}) \cdot v_{\lambda}^{*}=a^{\lambda} \cdot v_{\lambda}^{*}, \quad \operatorname{man} \in P=M A N .
$$

Here $a^{\lambda}=e^{\lambda(\log a)}$ as usual. In this case $\pi$ is said to be $(P, H)$-semispherical. Let

$$
f_{\pi}(g):=v_{\lambda}^{*}\left(\pi(g) v_{H}\right)
$$

be the corresponding matrix coefficient, then $f_{\pi}$ belongs to the space $\mathcal{P}_{+}$from Definition [3.5, and every element in $\mathcal{P}_{+}$is of this form for some $\pi$ and $v_{H}$. In particular, the fact that $Z$ is real spherical implies that $v_{\lambda}^{*}\left(v_{H}\right) \neq 0$, and this in turn implies that for a given character $\chi$ the semi-spherical vector $v_{H}$ is unique up to scaling.

Assume to begin with that $Z=G / H$ is a quasi-affine real spherical space. Let $\mathcal{P}_{+, 1} \subset \mathcal{P}_{+}$be the submonoid corresponding to right $H$ invariant functions, it then consists of the functions $f_{\pi}$ as above with 
trivial character $\chi$. Thus

$$
\mathcal{P}_{+, \mathbf{1}}=\mathbb{R}[G / H]^{M N}
$$

Set $A_{H}:=A \cap H$. In the sequel we prefer to consider $A_{Z}$ as a quotient $A_{Z}=A / A_{H}$. Let $(\pi, V)$ be an $H$-spherical irreducible representation as above. Then we define a cone $\mathfrak{a}_{Z, \pi}^{--} \subset \mathfrak{a}_{Z}$ by

$$
\mathfrak{a}_{Z, \pi}^{--}=\left\{X \in \mathfrak{a}_{Z} \mid \lim _{t \rightarrow \infty}\left[\pi(\exp (t X)) v_{H}\right]=\left[v_{-\lambda}\right] \in \mathbb{P}(V)\right\} .
$$

where $v_{-\lambda} \in V$ is a lowest weight vector.

Definition 5.1 (Compression cone). The closure $\mathfrak{a}_{Z, \pi}^{-}$of $\mathfrak{a}_{Z, \pi}^{-}$is called the compression cone of the $H$-spherical representation $\pi$. Taking the intersection over all $H$-spherical representations $\pi$ as above, the set

$$
\mathfrak{a}_{Z}^{-}=\bigcap_{\pi} \mathfrak{a}_{Z, \pi}^{-} \subseteq \mathfrak{a}_{Z}
$$

is called the compression cone of $Z$.

It will be seen later (below Lemma 5.6) that the intersection is finite. First we want to show that $\mathfrak{a}_{Z, \pi}^{-}$is a polyhedral convex cone. Let us expand the $H$-spherical vector $v_{H}$ into a-weights, say

$$
v_{H}=\sum_{\mu} v_{\mu}
$$

with $\pi(a) v_{\mu}=a^{\mu} \cdot v_{\mu}$ for $a \in A$. In particular, $v_{-\lambda} \neq 0$ since $v_{\lambda}^{*}\left(v_{H}\right) \neq 0$ and $v_{\lambda}^{*}\left(v_{\mu}\right)=0$ for $\mu \neq-\lambda$. We may assume (5.2) refers to this vector.

Remark 5.2. Note that $a^{\mu}=1$ for all $a \in A_{H}$ with $v_{\mu} \neq 0$.

Write $\Sigma_{\mathfrak{u}} \subset \Sigma^{+}$for the roots corresponding to $\mathfrak{u}$. As $V=\mathcal{U}(\mathfrak{u}) v_{-\lambda}$ it follows that all $\mathfrak{a}$-weights of $V$ are contained in $-\lambda+\mathbb{N}_{0}\left[\Sigma_{\mathfrak{u}}\right]$. Hence we find a finite subset $\Lambda_{\pi} \subset \mathbb{N}_{0}\left[\Sigma_{\mathfrak{u}}\right]$ such that

$$
v_{H}=\sum_{\nu \in \Lambda_{\pi}} v_{-\lambda+\nu}
$$

and $v_{-\lambda+\nu} \neq 0$ for $\nu \in \Lambda_{\pi}$. Since $v_{-\lambda} \neq 0$ we obtain:

Lemma 5.3. Let $(\pi, V)$ be an irreducible real $H$-spherical representation. Then for $X \in \mathfrak{a}_{Z}$ the following statements are equivalent:

(1) $X \in \mathfrak{a}_{Z, \pi}^{--}$

(2) $\nu(X)<0$ for all $\nu \in \Lambda_{\pi} \backslash\{0\}$.

In particular, $\mathfrak{a}_{Z, \pi}^{-}$is a finitely generated closed cone. 
Remark 5.4. Let $\mathfrak{a}^{--}$be the negative Weyl-chamber with respect to $\Sigma^{+}$, i.e.

$$
\mathfrak{a}^{--}:=\left\{X \in \mathfrak{a} \mid\left(\forall \alpha \in \Sigma^{+}\right) \alpha(X)<0\right\} .
$$

Then by the preceding lemma

$$
\left(\mathfrak{a}^{--}+\mathfrak{a}_{H}\right) / \mathfrak{a}_{H} \subseteq \mathfrak{a}_{Z, \pi}^{--} .
$$

There are various instances when the inclusion (5.4) is strict. For example, if $\pi$ is the trivial representation. A more serious obstruction will be encountered in the next section when we discuss wave front spherical spaces.

Let us now describe how the $\mathfrak{a}_{Z, \pi}^{-}$behave under tensor products. For notational reasons we prefer to write $\mathfrak{a}_{Z, \lambda}^{-}$instead of $\mathfrak{a}_{Z, \pi}^{-}$with $-\lambda$ being the lowest weight of $(\pi, V)$. Let $\pi, \pi^{\prime}$ be two irreducible representations as above with lowest weights $-\lambda$ and $-\lambda^{\prime}$, and with $H$-fixed vectors $v_{H}$ and $v_{H}^{\prime}$. Then $\pi \otimes \pi^{\prime}$ has a lowest weight vector $v_{-\lambda} \otimes v_{-\lambda^{\prime}}^{\prime}$ and an $H$-spherical vector $v_{H} \otimes v_{H}^{\prime}$. Let $(\rho, W)$ be the irreducible subrepresentation of $\pi \otimes \pi^{\prime}$ which is generated by the lowest weight vector $v_{-\lambda} \otimes v_{-\lambda^{\prime}}^{\prime}$. Write $p: V \otimes V^{\prime} \rightarrow W$ for the $G$-equivariant projection. Then $0 \neq w_{H}:=p\left(v_{H} \otimes v_{H}^{\prime}\right)$ is an $H$-fixed vector of $W$.

Proposition 5.5. With the notation introduced above the following holds:

$$
\mathfrak{a}_{Z, \lambda+\lambda^{\prime}}^{-}=\mathfrak{a}_{Z, \lambda}^{-} \cap \mathfrak{a}_{Z, \lambda^{\prime}}^{-}
$$

Proof. The inclusion $\supseteq$ is clear from Lemma 5.3 and the relation $\Lambda_{\rho} \subseteq$ $\Lambda_{\pi}+\Lambda_{\pi^{\prime}}$. To obtain the opposite inclusion let us call an element $\mu \in$ $\Lambda_{\pi} \cup \Lambda_{\pi^{\prime}}$ indecomposable if it cannot be expressed as $\mu=\mu_{1}+\mu_{2}$ with non-zero elements $\mu_{i} \in \Lambda_{\pi} \cup \Lambda_{\pi^{\prime}}$. Thus the opposite inclusion will follow provided that $\mu \in \Lambda_{\rho}$ for all indecomposable $\mu$. Without loss of generality let $\mu \in \Lambda_{\pi}$.

Note that the matrix coefficient $\left(g \cdot\left(v_{\lambda}^{*} \otimes v_{\lambda^{\prime}}^{*}\right)\right)\left(v_{-\lambda+\mu} \otimes v_{-\lambda^{\prime}}^{\prime}\right)$ is the product of nonzero matrix coefficients $\left(g \cdot v_{\lambda}^{*}\right)\left(v_{-\lambda+\mu}\right)$ and $\left(g \cdot v_{\lambda^{\prime}}\right)\left(v_{-\lambda^{\prime}}^{\prime}\right)$. Hence we can select $g \in G$ such that

$$
\left(g \cdot\left(v_{\lambda}^{*} \otimes v_{\lambda^{\prime}}^{*}\right)\right)\left(v_{-\lambda+\mu} \otimes v_{-\lambda^{\prime}}^{\prime}\right) \neq 0 .
$$

We consider the following algebraic function $F$ on $A$ :

$$
F(a)=\left(g \cdot\left(v_{\lambda}^{*} \otimes v_{\lambda^{\prime}}^{*}\right)\right)\left(a \cdot w_{H}\right)=\left(g \cdot\left(v_{\lambda}^{*} \otimes v_{\lambda^{\prime}}^{*}\right)\right)\left(a \cdot\left(v_{H} \otimes v_{H}^{\prime}\right)\right) .
$$

Then $F$ is a linear combination of characters on $A$. As $\mu$ was indecomposable, (5.6) implies that the coefficient of $a^{-\lambda-\lambda^{\prime}+\mu}$ in the expansion of $F$ is non-zero. Hence $\mu \in \Lambda_{\rho}$ and the proof is complete.

In order to move on we need: 
Lemma 5.6. The multiplicative monoid $\mathcal{P}_{+, 1}$ is finitely generated.

Proof. Recall (5.1) and observe that

$$
\mathbb{R}[G / H]^{M N} \simeq \mathbb{R}[G / M N \times Z]^{G} .
$$

Further $G / M N$ is quasi-affine and hence so is $G / M N \times Z$. As $G$ is algebraic real reductive we conclude from Hilbert's theorem that $\mathbb{R}[G / M N \times Z]^{G}$ is finitely generated.

Let $f_{1}, \ldots, f_{n}$ be a set of generators of $\mathcal{P}_{+, 1}$ and let $f_{i}=f_{\pi_{i}}$. Then Proposition 5.5 implies that $\mathfrak{a}_{Z}^{-}=\cap_{i} \mathfrak{a}_{Z, \pi_{i}}^{-}$. Moreover, we have:

Lemma 5.7. Let $f=f_{\pi} \in \mathcal{P}_{++, 1}$. Then

$$
\mathfrak{a}_{Z}^{-}=\mathfrak{a}_{Z, \pi}^{-} .
$$

Proof. As in 4, Lemme 2.1, we use that $\mathbb{C}[G]$ is a Krull domain to conclude that the product $f_{1} \cdots f_{n}$ divides $f^{N}$ for some $N \in \mathbb{N}$. The result then follows from Proposition 5.5.

So far we have assumed that $Z$ is quasi-affine. The general case is reduced to this case as follows.

One needs to allow the vector $v_{H}=v_{H, \chi}$ to transform under the character $\chi$ of $H$. Let

$$
f_{\pi, \chi}(g):=v_{\lambda}^{*}\left(\pi(g) v_{H, \chi}\right) .
$$

As before we expand

$$
v_{H, \chi}=\sum_{\mu \in \Lambda_{\pi, \chi}} v_{-\lambda+\mu}
$$

and observe that each $\mu \in \Lambda_{\pi, \chi}$ is trivial on $\mathfrak{a}_{H}$, so that $\Lambda_{\pi, \chi} \subset \mathfrak{a}_{Z}^{*}$. As in (5.2) one defines an open cone $\mathfrak{a}_{Z, \pi, \chi}^{-}$, and taking closures one defines

$$
\mathfrak{a}_{Z, \chi}^{-}=\bigcap_{\pi} \mathfrak{a}_{Z, \pi, \chi}^{-}, \quad \mathfrak{a}_{Z}^{-}=\bigcap_{\chi} \mathfrak{a}_{Z, \chi}^{-}=\bigcap_{\pi, \chi} \mathfrak{a}_{Z, \pi, \chi}^{-}
$$

as in Definition 5.1

For each $\chi$ we define the quasi-affine space $Z_{1, \chi}$ as in (2.2), then $\mathfrak{a}_{Z_{1, \chi}}^{-}=\mathfrak{a}_{Z, \chi}^{-} \times \mathbb{R}$. From our discussion of the quasi-affine situation we deduce that the first intersection above is finite and that

$$
\mathfrak{a}_{Z, \chi}^{-}=\mathfrak{a}_{Z, \pi, \chi}^{-} \quad \text { if } \quad f_{\pi, \chi} \in \mathcal{P}_{++} .
$$

It remains to be seen that $\mathfrak{a}_{Z, \chi}$ is independent of $\chi$. We employ the notation before Proposition 5.5 and note that $f_{\pi, \chi} \cdot f_{\pi^{\prime}, \chi^{\prime}}=f_{\rho, \chi \chi^{\prime}}$. As in Proposition 5.5 we deduce that $\mathfrak{a}_{Z, \pi, \chi}^{-} \cap \mathfrak{a}_{Z, \pi^{\prime}, \chi^{\prime}}^{-}=\mathfrak{a}_{Z, \rho, \chi \chi^{\prime}}^{-}$. In particular 
if both $f_{\pi, \chi}$ and $f_{\pi^{\prime}, \chi^{\prime}}$ are in $\mathcal{P}_{++}$, then we obtain with (5.8) that $\mathfrak{a}_{Z, \chi}^{-} \cap$ $\mathfrak{a}_{Z, \chi^{\prime}}^{-}=\mathfrak{a}_{Z, \chi \chi^{\prime}}$. Proceeding as in the quasiaffine case we get

$$
\mathfrak{a}_{Z}^{-}=\mathfrak{a}_{Z, \pi, \chi}^{-} \quad \text { if } \quad f_{\pi, \chi} \in \mathcal{P}_{++} .
$$

5.2. The edge of the compression cone. Let $C$ be a closed convex cone in a finite dimensional real vector space $V$. The edge of $C$ is the linear subspace $E(C):=C \cap-C$ of $V$. One calls $C$ pointed or sharp provided that $E(C)=\{0\}$.

We deduce from Lemma 3.12 the direct sum decomposition for the Lie algebra of $N_{G}(\mathfrak{h})$ :

$$
\mathfrak{n}_{\mathfrak{g}}(\mathfrak{h})=\mathfrak{h} \oplus \tilde{\mathfrak{a}}_{h} \oplus \tilde{\mathfrak{m}}_{h}
$$

with subspaces $\tilde{\mathfrak{a}}_{h} \subset \mathfrak{a}$ and $\tilde{\mathfrak{m}}_{h} \subset \mathfrak{m}$.

Lemma 5.8. (Edge of the Compression cone) The following assertions are equivalent:

(1) $\tilde{\mathfrak{a}}_{h}=\{0\}$.

(2) $N_{G}(\mathfrak{h}) / H$ is compact.

(3) $\mathfrak{a}_{Z}^{-}$is sharp.

Proof. The equivalence of (1) and (2) follows from (5.10).

By the definition of the compression cone we have

$$
\tilde{\mathfrak{a}}_{h}+\mathfrak{a}_{Z, \pi, \chi}^{--}=\mathfrak{a}_{Z, \pi, \chi}^{--}
$$

for each $\pi$ and $\chi$, and hence $\tilde{\mathfrak{a}}_{h} \subset \mathfrak{a}_{Z}^{-}$. Thus if $\tilde{\mathfrak{a}}_{h} \neq\{0\}$, then $\mathfrak{a}_{Z}^{-}$is not sharp, i.e. (3) implies (1). Finally, it follows from Lemma 5.3 that the edge $E\left(\mathfrak{a}_{Z}^{-}\right)$fixes the line $\mathbb{R} v_{H, \chi}$. Hence $E\left(\mathfrak{a}_{Z}^{-}\right) \subset \mathfrak{j}$ (see Lemma 3.9), and then $E\left(\mathfrak{a}_{Z}^{-}\right) \subset \mathfrak{n}_{\mathfrak{g}}(\mathfrak{h})$ since $\mathfrak{j} / \mathfrak{n}_{\mathfrak{g}}(\mathfrak{h})$ has compact Lie algebra (see Theorem 3.16). Thus (1) implies that $E\left(\mathfrak{a}_{Z}^{-}\right) \subset \mathfrak{h}$ and (3) holds.

5.3. Compression in the Grassmannian. We define the limiting subalgebra

$$
\mathfrak{h}_{\lim }=\overline{\mathfrak{u}}+(\mathfrak{l} \cap \mathfrak{h}) .
$$

From (2.4) we obtain that $\mathfrak{h}_{\text {lim }}$ is a real spherical subalgebra of $\mathfrak{g}$ with

$$
d:=\operatorname{dim} \mathfrak{h}=\operatorname{dim} \mathfrak{h}_{\text {lim }} .
$$

Let us denote by $\operatorname{Gr}_{d}(\mathfrak{g})$ the Grassmannian of $d$-dimensional subspaces of the real vector space $\mathfrak{g}$.

Denote by $\mathfrak{a}_{Z}^{--}$the interior of $\mathfrak{a}_{Z}^{-}$. The following result is motivated by the work of Brion, see [3], Section 2 .

Lemma 5.9. Let $X \in \mathfrak{a}_{Z}$. Then the following statements are equivalent: 
(1) $X \in \mathfrak{a}_{Z}^{--}$.

(2) $\lim _{t \rightarrow \infty} e^{t \operatorname{ad} X} \mathfrak{h}=\mathfrak{h}_{\text {lim }}$ in $\operatorname{Gr}_{d}(\mathfrak{g})$.

Proof. Let $X \in \mathfrak{a}_{Z}$. Set $V:=\bigwedge^{d} \mathfrak{g}$ and endow $V$ with an inner product $\langle\cdot, \cdot\rangle$ which is $\theta$-covariant, that is: $\langle g \cdot v, w\rangle=\left\langle v, \theta(g)^{-1} \cdot w\right\rangle$ for all $v, w \in V$ and $g \in G$.

Let $v_{0}, v_{H} \in V$ be lifts of $\mathfrak{h}_{\text {lim }}, \mathfrak{h} \in \mathrm{Gr}_{d}(\mathfrak{g})$ to $V$. Note that $v_{0}$ is an eigenvector for $A \bar{N}$ with a-weight $-2 \rho_{\mathfrak{u}}:=-\sum_{\alpha \in \Sigma_{\mathfrak{u}}} \alpha$. Denote by $W$ the $G$-submodule of $V$ which is generated by $v_{0}$ and write $p: V \rightarrow W$ for the orthogonal projection. Set $w_{H}:=p\left(v_{H}\right)$ and $w_{0}:=p\left(v_{0}\right)=v_{0}$. Then we need to show that:

$$
X \in \mathfrak{a}_{Z}^{--} \Longleftrightarrow \lim _{t \rightarrow \infty}\left[\exp (t X) \cdot w_{H}\right]=\left[w_{0}\right]
$$

in $\mathbb{P}(V)$.

Let $W^{*}$ be the dual representation of $W$ and $w_{0}^{*} \in W^{*}$ be a functional with $a n \cdot w_{0}^{*}=a^{2 \rho_{\mathfrak{u}}} w_{0}^{*}$ for all $a n \in A N$ and $w_{0}^{*}\left(w_{0}\right) \neq 0$. Attached to $w_{0}^{*}$ and $w_{H}$ is the $A N \times H$-semi-invariant function

$$
F(g):=w_{0}^{*}\left(g \cdot w_{H}\right) .
$$

In general this is not a left $P$-eigenfunction, but we can overcome this difficulty by passing to the $M$-average of the square as in the proof of Lemma 3.14:

$$
f(g):=\int_{M} F(m \cdot g)^{2} d m \quad(g \in G) .
$$

Then $f$ is a matrix coefficient of a finite dimensional irreducible representation $U$ with $H$-fixed vector $u_{H}$ and a $P$-eigenvector $u_{0}^{*} \in U^{*}$ with $\operatorname{man} \cdot u_{0}^{*}=a^{4 \rho_{\mathfrak{u}}} u_{0}^{*}$ :

$$
f(g)=u_{0}^{*}\left(g \cdot u_{H}\right) \quad(g \in G) .
$$

As $4 \rho_{\mathfrak{u}}\left(\alpha^{\vee}\right)>0$ for all $\alpha \in \Sigma_{\mathfrak{u}}$ we conclude that $f \in \mathcal{P}_{++}$. From (5.7) we thus conclude that $X \in \mathfrak{a}_{Z}^{--}$if and only if

$$
\lim _{t \rightarrow \infty}\left[\exp (t X) \cdot u_{H}\right]=\left[u_{0}\right]
$$

holds true. Hence it remains to be seen that (5.13) is equivalent to the right hand side of (5.11).

For that let $w_{H}=\sum_{\nu \in \Lambda_{F}} v_{-2 \rho_{\mathfrak{u}}+\nu}$ and $u_{H}=\sum_{\nu \in \Lambda_{f}} u_{-4 \rho_{\mathfrak{u}}+\nu}$ be the respective decomposition into non-trivial a-weight vectors, then $\Lambda_{f} \subseteq \Lambda_{F}+\Lambda_{F}$, and hence (5.13) is implied by the right hand side of (5.11). For the converse implication, we observe that if $\nu \in \Lambda_{F}$ is indecomposable (that is, not the sum of two non-zero elements from $\Lambda_{F}$ ), then $2 \nu \in \Lambda_{f}$. This follows from the construction (5.12), as in 
the proof of Proposition 5.5. The implication is an easy consequence of this observation.

5.4. An explicit description of the compression cone. This part is an adaption of Section 2.3 from [3].

Write $\mathfrak{d}_{H}^{\perp}$ for the orthocomplement of $\mathfrak{d}_{H}:=\mathfrak{d} \cap \mathfrak{h}$ in $\mathfrak{d}$. The local structure theorem implies that

$$
\mathfrak{h}=\mathfrak{l} \cap \mathfrak{h} \oplus \mathcal{G}(T)
$$

where $\mathcal{G}(T)$ is the graph of a linear map

$$
T: \overline{\mathfrak{u}} \rightarrow \mathfrak{u}+\mathfrak{d}_{H}^{\perp} .
$$

In particular, for all $\alpha \in \Sigma_{\mathfrak{u}}$ and root vectors $X_{-\alpha} \in \mathfrak{g}^{-\alpha}$ we find $D_{\alpha} \in \mathfrak{d}_{H}^{\perp}$ and $X_{\beta} \in \mathfrak{g}^{\beta}, \beta \in \Sigma_{\mathfrak{u}}$ such that

$$
Y_{\alpha}:=X_{-\alpha}+D_{\alpha}+\sum_{\beta} X_{\beta} \in \mathfrak{h} .
$$

As $\left[\mathfrak{a}_{H}, Y_{\alpha}\right] \in \mathcal{G}(T)$ we conclude that: $\left.\alpha\right|_{\mathfrak{a}_{H}}=0$ if $D_{\alpha} \neq 0$, and $(\alpha+$ $\beta)\left.\right|_{\mathfrak{a}_{H}}=0$ if $X_{\beta} \neq 0$. We let $\mathcal{M} \subset \mathfrak{a}_{Z}^{*} \simeq \mathfrak{a}_{H}^{\perp} \subset \mathfrak{a}^{*}$ be the monoid, i.e. additive semi-group, generated by

- $\alpha$ if there exists $X_{-\alpha}$ with $D_{\alpha} \neq 0$.

- $\alpha+\beta$ if there exists $X_{-\alpha}$ with $X_{\beta} \neq 0$.

We combine Lemma 5.3 with Lemma 5.9 and obtain that:

Lemma 5.10. $\mathfrak{a}_{Z}^{-}=\left\{X \in \mathfrak{a}_{Z} \mid(\forall \alpha \in \mathcal{M}) \alpha(X) \leq 0\right\}$.

Remark 5.11. In the case where $Z_{\mathbb{C}}$ is spherical, the compression cone coincides with the so-called valuation cone. This follows from Lemma 5.10 in combination with [3], Cor. 2.4.

5.5. Refined Polar decomposition. Set $A_{Z}^{-}:=\exp \mathfrak{a}_{Z}^{-} \subseteq A / A_{H}$.

Proposition 5.12. Let $Z=G / H$ be a real spherical space with $H=J$ and simple compactification $\widehat{Z}$ as in Section 4. Then the map

$$
\Phi: K \times \overline{A_{Z}^{-} F \cdot z_{0}} \rightarrow \widehat{Z}, \quad(k, s) \mapsto k \cdot s
$$

is open in $(\mathbf{1}, \widehat{z})$. In particular one has $G=F^{\prime} K A_{Z}^{-} F H$ for a finite set $F^{\prime} \subset G$.

Proof. Let $(\pi, V)$ be an $H$-semi-spherical representation out of which we constructed $\widehat{Z} \subset \mathbb{P}(V)$. We use that $\mathfrak{a}_{Z}^{-}$is the compression cone of $\pi$, see Lemma 5.7 and the discussion below it. Note that $z_{0}=\left[v_{H}\right]$ and $\widehat{z}=\left[v_{0}\right]$. We claim that $\overline{M A_{Z}^{-} F \cdot z_{0}}$ is a neighborhood of $\widehat{z}$ in the slice. In fact, by the definition of the compression cone we see that $T_{Z}\left(A_{Z} \backslash A_{Z}^{-}\right) \cdot z_{0}$ does not meet a neighborhood of $\widehat{z}$. The claim follows. 
By Lemma 4.2 the map $\Phi$ is open and the assertion follows as in the proof of Lemma 4.1 .

As in Section 4 we obtain as a corollary:

Theorem 5.13. (Fine Polar decomposition) Suppose that $Z=G / H$ is a real spherical space. Then there exists a compact subset $\Omega \subset G$ and a finite set $F^{\prime \prime} \subset G$ such that

$$
G=\Omega A_{Z}^{-} F^{\prime \prime} H \text {. }
$$

Moreover, one has:

(1) $\Omega=F^{\prime} K$ for some finite set $F^{\prime} \subset G$.

(2) $F^{\prime \prime} \subseteq F N_{G}(H)$.

Example 5.14. In many examples it appears that $F^{\prime \prime}$ can be taken in $N_{G}(H)$. However it cannot be skipped completely as the basic example $Z=\mathrm{SL}(2, \mathbb{R}) / \mathrm{SO}(1,1)$ shows. Let $A$ be the diagonal matrices in $G=$ $\mathrm{SL}(2, \mathbb{R})$ with positive entries and $K=\mathrm{SO}(2, \mathbb{R})$. We realize $Z$ as the one sheeted hyperboloid in $\mathbb{R}^{3}$ :

$$
Z=\left\{(x, y, z) \in \mathbb{R}^{3} \mid x^{2}+y^{2}-z^{2}=1\right\}
$$

with base point $z_{0}=(1,0,0)$. Then

$$
A_{Z}^{-} \cdot z_{0}=A^{-} \cdot z_{0}=\{(x, 0, z) \in Z \mid x, z \geq 0\} .
$$

Hence it it is not possible that $Z=\Omega A_{Z}^{-} \cdot z_{0}$ holds for a compact set $\Omega \subset G$. Here $N_{G}(H)_{0}=H$ and the quotient $N_{G}(H) / H$ is realized by the involutive element $w=\left(\begin{array}{cc}0 & 1 \\ -1 & 0\end{array}\right) \in K$ which satisfies $w A_{Z}^{-} w=A_{Z}^{+}$. With $\Omega=K$ one then has $G=K A_{Z} H$.

\section{WAVEFRONT SPHERICAL SPACES AND THE WAVEFRONT LEMMA}

Denote by $\mathfrak{a}^{-}$the closure of the negative Weyl chamber $\mathfrak{a}^{--}$. The following definition is motivated by [16].

Definition 6.1. We call the real spherical space $Z=G / H$ wavefront provided that

$$
\mathfrak{a}_{Z}^{-}=\left(\mathfrak{a}^{-}+\mathfrak{a}_{H}\right) / \mathfrak{a}_{H} .
$$

Remark 6.2. All symmetric spaces are wavefront. Moreover, if $G$ and $H$ are complex then one can decide with the Luna diagram whether $Z=G / H$ is wavefront. For example all complex spherical spaces of the type $Z=G \times H / \operatorname{diag}(H)$ are wavefront. A few others, such as $\mathrm{SO}(2 n+1, \mathbb{C}) / \mathrm{GL}(n, \mathbb{C}), \mathrm{GL}(2 n+1, \mathbb{C}) / \mathrm{Sp}(2 n, \mathbb{C})$ or $\mathrm{SO}(8, \mathbb{C}) / G_{2}$ are not wavefront. To be precise: from the 78 cases in the list of [2], the non-wavefront cases are: (11), (24), (25), (27), (39-50), (60) and (61). 
Denote by $A^{-}$the closure of the negative Weyl chamber with respect to the positive system determined by $N$. Notice that (6.1) implies that

$$
A_{Z}^{-} \cdot z_{0}=A^{-} \cdot z_{0}
$$

With that we obtain a generalization of the "wavefront lemma" of Eskin-McMullen ([7] Theorem 3.1). The technique of the proof is essentially known - see for instance [16] or [13].

Lemma 6.3. Suppose that $Z=G / H$ is a wavefront real spherical space. Then there exists a closed subset $E \subset G$ with the following properties.

(1) $E \rightarrow G / H$ is surjective.

(2) For every neighborhood $\mathcal{V}$ of $\mathbf{1}$ in $G$, there exists a neighborhood $\mathcal{U}$ of $\mathbf{1}$ in $G$ such that

$$
\mathcal{V} g \cdot z_{0} \supset g \mathcal{U} \cdot z_{0}
$$

for all $g \in E$.

Proof. Put

$$
E=\Omega A^{-} F^{\prime \prime}
$$

Then (1) follows from (6.2) and Corollary 5.13.

The proof of (2) is similar to [13], Lemma 5.4. For the convenience to the reader we recall the argument.

For a compact set $\Omega \subset G$ we note that the set $\bigcap_{x \in \Omega} \operatorname{Ad}\left(x^{-1}\right) \mathcal{V}$ is a neighborhood of $\mathbf{1}$ in $G$. Then the assertion is reduced to the case where $g \in A^{-} F^{\prime \prime}$.

Let $\mathcal{U}_{1}$ be a neighborhood of $\mathbf{1}$ in $P$ which is contained in $\mathcal{V}$ and which is stable under conjugation by elements from $A^{-}$. As $P f H$ is open for all $f \in F^{\prime \prime}$, we see that $f^{-1} \mathcal{U}_{1} f \cdot z_{0}$ is a neighborhood of $z_{0}$ for each $f \in F^{\prime \prime}$. We choose $\mathcal{U}$ so small that

$$
\mathcal{U} \cdot z_{0} \subset f^{-1} \mathcal{U}_{1} f \cdot z_{0}
$$

holds for all $f \in F^{\prime \prime}$. Then for $g=a f$ with $a \in A^{-}$we obtain

$$
a f \mathcal{U} \cdot z_{0} \subset a \mathcal{U}_{1} f \cdot z_{0} \subset \mathcal{U}_{1} a f \cdot z_{0} \subset \mathcal{V} a f \cdot z_{0} .
$$

Remark 6.4. Lemma 6.3 suggests that real spherical spaces which are wavefront are especially suited to discuss the lattice counting problem, see [7]. Having developed the harmonic analysis on real spherical spaces further one can obtain error term bounds for the lattice counting problem. We will return to this topic in an upcoming publication. 


\section{REFERENCES}

[1] F. Bien, Orbits, multiplicities and differential operators. Representation theory of groups and algebras, 199-227, Contemp. Math. 145, Amer. Math. Soc., Providence, RI, 1993.

[2] P. Bravi and G. Pezzini, The spherical systems of the wonderful reductive subgroups, arXiv:1109.6777

[3] M. Brion, Vers une généralisation des espaces symétriques, J. Alg. 134 (1990), 115-143.

[4] M. Brion, D. Luna and Th. Vust, Espaces homogènes sphériques, Invent. Math. 84 (1986), no. 3, 617-632.

[5] M. Brion and F. Pauer, Valuations des espaces homogènes sphériques, Comment. Math. Helv. 62 (1987), no. 2, 265-285.

[6] T. Danielsen, B. Krötz and H. Schlichtkrull, Decomposition theorems for triple spaces, arXiv:1301.0489

[7] A. Eskin and C. McMullen, Mixing, counting, and equidistribution in Lie groups, Duke Math. J. 71 (1993), no. 1, 181-209.

[8] J. Goodman, Affine open subsets of algebraic varieties and ample divisors Ann. of Math. (2) 89 (1969), 160-183.

[9] F. Knop, Automorphisms, root systems, and compactifications of homogeneous varieties, J. Amer. Math. Soc. 9 (1996), no. 1, 153-174.

[10] F. Knop, H. Kraft, D. Luna and T. Vust, Local properties of algebraic group actions. Algebraische Transformationsgruppen und Invariantentheorie, 63-75, DMV Sem. 13, Birkhäuser, Basel, 1989.

[11] F. Knop, H. Kraft and T. Vust, The Picard group of a G-variety. Algebraische Transformationsgruppen und Invariantentheorie, 77-87, DMV Sem. 13, Birkhäuser, Basel, 1989.

[12] F. Knop, B. Krötz and H. Schlichtkrull, The local structure theorem for real spherical spaces, arXiv: 1310.6390

[13] B. Krötz, E. Sayag and H. Schlichtkrull, Decay of matrix coefficients on reductive homogeneous spaces of spherical type, arXiv: 1211.2943. To appear in Math. Z.

[14] B. Krötz and H. Schlichtkrull, Finite orbit decomposition of real flag manifolds, arXiv:1307.2375

[15] Multiplicity bounds and the subrepresentation theorem for real spherical spaces, arXiv:1309.0930, to appear in Trans. AMS

[16] Y. Sakellaridis and A. Venkatesh, Periods and harmonic analysis on spherical varieties, arXiv:1203.0039 